\title{
Special issue on naming species, ranking and evolution of fungi
}

\author{
Hongsanan $\mathbf{S}^{1 *}$, Sánchez-Ramírez $\mathrm{S}^{2,3}$, Zhao $\mathrm{RL}^{4}$ \\ ${ }^{1}$ Center of Excellence in Fungal Research, Chiang Rai 57100, Thailand \\ ${ }^{2}$ Department of Natural History, Royal Ontario Museum, Toronto, ON M5S 2C6, Canada \\ ${ }^{3}$ Department of Ecology and Evolutionary Biology, University of Toronto, Toronto, ON M5S 3B2, Canada \\ ${ }^{4}$ State Key Laboratory of Mycology, Institute of Microbiology, Chinese Academy of Sciences, Beijing 100101, People's \\ Republic of China \\ E-mail: <sinang333@gmail.com>
}

Hongsanan S, Sánchez-Ramírez S, Zhao RL. 2016 - Special issue on naming species, ranking and evolution of fungi. Mycosphere 7 (11), 1620-1621, Doi 10.5943/mycosphere/7/11/1

The classification and taxa delimitation in the fungi are often subjective, being based on morphological differences and therefore other studies (e.g. phylogenetics) are needed to clarify unresolved taxa. The ranking of fungal species especially at the higher levels needs sets of evidence for their delimitation. Morphology and molecular data have been used for fungal identification and classification for many years. However, there are many problematic groups that need further collections and evidence and new techniques for their clarification. Many species also need to be restudied since they have been introduced repeatedly from different parts of the world. Thus, giving wrong name can cause many problems in identification and classification.

The molecular dating technique is a key tool for interpreting the history of life of any organisms and it presently has become a common tool for many biologists in research areas. The technique has developed considerably over the last 35 years, and now with these developments the molecular dating technique is becoming more statistical accurate and can provide an extensive heterogeneity of evolutionary rates. Molecular clock analysis can use information from anatomy/ embryology, biogeography, fossils and biological molecules. Fungal evolutionary study has used fossil evidence and molecular data to estimate the evolutionary events. Many thousands of papers have used molecular dating for estimating species divergences times. The evolutionary events can explain the rate of mutation of the interested organisms and present their history of life. The major factor that can cause mutation in each evolutionary can be estimated. Presently, researchers have increasingly used larger datasets that can estimate a much greater diversity of species.

This special issue of Mycosphere comprises eight papers that represent naming species, ranking and evolution of fungi. The first paper by Dayarathne et al. discussed the taxonomic utility of using old names in current fungal classification and nomenclature. This paper provides potential solutions to facilitate future naming and classification of fungi. The second paper by Liu et al. discusses the concepts of genus, family and order, including definitions and various ways of that ranks has been used in the fungi. Jeewon et al. (Paper 3) discusses establishing species boundaries and new taxa among fungi: recommendations to resolve taxonomic ambiguities is presented. Samarakoon et al. (Paper 4) compares the divergence and ranking of taxa across the kingdoms Animalia, Fungi and Plantae. Hongsanan et al. (Paper 5) studied the evolution of fungal epiphytes within Dothideomycetes and Sordariomycetes, providing divergence time estimates. Phukhamsakda 
et al. (Paper 6) provides the evolutionary history of Massarineae members, and they introduce the new family Longipedicellataceae using additional evidence from molecular dating analysis. Moreover, the same technique is used to confirm the relationship between Muyocopronales and a new family, Palawaniaceae, introduced by Mapook et al. (Paper 7). The study on the evolution of Xylariomycetidae (Ascomycota: Sordariomycetes) is provided by Samarakoon et al. (Paper 8). The authors compare the results from molecular dating to that from phylogenetics. 